\title{
Applications of Nanotechnology in the Treatment of Heart Attack and Cancer
}

\author{
K.Sonamuthu \\ Department of Physics ,J.N.R.M., Port Blair, South Andaman
}

\begin{abstract}
Nanotechnology is unique in that it represents not just one specific area, but a vast variety of disciplines ranging from basic material science to personal care applications. Thus this technology is very advanced and various researches are a heading in this field very quickly One of the important areas of nanotechnology is "nanomedicine," which, refers to highly specific medical intervention at the molecular scale for diagnosis, prevention and treatment of diseases. The importance of nanotechnology in drug delivery is in the concept and ability to manipulate molecules and super molecular structures for producing devices with programmed functions. Conventional liposomes, polymeric micelles, and nanoparticles are now called "nanovehicles,". Those conventional drug delivery systems would have evolved to the present state regardless of the current nanotechnology revolution. To achieve efficient drug delivery it is important to understand the interactions of nanomaterials with the biological environment, targeting cell-surface receptors, drug release, and molecular mechanisms of cell signalling involved in pathobiology of the disease under consideration. Several anti-cancer drugs including paclitaxel, doxorubicin, 5fluorouracil and dexamethasone have been successfully formulated using nanomaterials. The nano drug delivery systems hold great potential to overcome some of the barriers of cells and molecules in heart attack and cancer.

Keywords: Nanotechnology, nanomedicine, nanoparticles, liposomes, polymeric micelles, nanoparticles, dendrimers, and nanocrystals. Liposomes and polymer micelles, PLGA, etc,.
\end{abstract}

DOI: $10.7176 /$ APTA/80-03

Publication date:October $31^{\text {st }} 2019$

\section{INTRODUCTION}

Living organisms contain many molecules which interact with nanoparticles. Most often, nanomaterials will become coated with protein molecules. This "corona" of proteins is a shifting population of different molecules. Key factors in the interaction with living structures include nanoparticle dose, the ability of nanoparticles to spread within the body, as well as their solubility. Some nanoparticles dissolve easily and their effects on living organisms are the same as the effects of the chemiotheraphy.

The reason why these nanoparticles are attractive for medical purposes is based on their important and unique features, such as their surface to mass ratio that is much larger than that of other particles, their quantum properties and their ability to adsorb and carry other compounds. nanoparticles have a relatively large functional surface which is able to bind, adsorb and carry other compounds such as drugs, probes and proteins. However, many challenges must be overcome if the application of nanotechnology is to realize the anticipated improved understanding of the patho-physiological basis of disease, bring more sophisticated diagnostic opportunities, and yield improved therapies. Nanoparticles hold tremendous potential as an effective drug delivery system. To achieve efficient drug delivery it is important to understand the interactions of nanomaterials with the biological environment, targeting cell-surface receptors, drug release, and molecular mechanisms of cell signalling involved in pathobiology of the disease under consideration. Several anti-cancer drugs including paclitaxel, doxorubicin, 5fluorouracil and dexamethasone have been successfully formulated using nanomaterials. Quantom dots, chitosan, Polylactic/glycolic acid (PLGA) based nanoparticles have been used Anti-cancer drugs . Brain cancer is one of the most difficult malignancies to detect and treat mainly because of the difficulty in getting imaging and therapeutic agents past the blood-brain barrier and into the brain. Anti-cancer drugs such as loperamide and doxorubicin bound to nanomaterials and released at therapeutic concentrations in the brain.

\section{Preparation of Nanoparticles through ionic gelation method}

Nanoparticles can be prepared from a variety of materials such as proteins, polysaccharides and synthetic polymers. The selection of matrix materials is dependent on many factors including (a) size of nanoparticles required; (b) inherent properties of the drug and stability; (c) surface characteristics such as charge and permeability; (d) degree of biodegradability and toxicity and (f) Antigenicity of the final product. Nanoparticles have been prepared most frequency by three methods: (1) dispersion of preformed polymers; (2) polymerization of monomers; and (3) ionic gelation or coacervation of hydrophilic polymers. Here, the ionic gelation method has been focused on the preparation of nanoparticles using biodegradable hydrophilic polymers such as chitosan, gelatin and sodium alginate. The method involves a mixture of two aqueous phases, of which one is the polymer chitosan, a di-block co-polymer ethylene oxide or propylene oxide (PEO-PPO) and the other is a polyanion sodium tripolyphosphate. In this method, positively charged amino group of chitosan interacts with negative charged tripolyphosphate to 
form coacervates with a size in the range of nanometer. Coacervates are formed as a result of electrostatic interaction between two aqueous phases, whereas, ionic gelation involves the material undergoing transition from liquid to gel due to ionic interaction conditions at room temperature.

\section{DISCUSSION}

\subsection{Interaction of nanoparticles with human cancer cell.}

Cancer is one of the most challenging diseases today, and brain cancer is one of the most difficult malignancies to detect and treat mainly because of the difficulty in getting imaging and therapeutic agents across the blood-brain barrier and into the brain. Apolipoprotein E was suggested to mediate drug transport across the blood-brain barrier Loperamide, which does not cross the blood-brain barrier but exerts antinociceptive effects after direct injection into the brain, was loaded into human serum.

Nanoshells may be used to concentrate the heat from infrared light to kill cancer cells with minimal damage to surrounding healthy body cells. A nanoshell consists of a gold coated metal shell and a non-conducting silica centre and the shell is able to absorb nearby infrared laser energy emitted by a source. The nanoparticles are injected into the bloodstream where they travel to the cancerous growth. The advantage of using nanoshells to treat cancerous tumours is that they can destroy cancerous cells but do not excessively damage normal cells in the process (see Figure 2). There is an advantage over chemotherapy, which destroys both cancerous and normal cells. The drugs used in chemotherapy are not able to target the cancerous growth specifically, and the damage of healthy body cells can result in secondary conditions such as immune system suppression and hair loss, whereas nanoparticles have the potential to be able to target only the cancer cells, so prove to be a more harmless treatment.

Quantum Dots may be used in the future for locating cancer tumours in patients. where the quantum dots have been observed to give off a detectable fluorescence when stimulated by UV light, hence indicating the position of the tumour. By combining different sized quantum dots within individual granules, granules that release distinctive colours and intensities of light, after stimulation by UV light, can be created. The large variety of quantum dots can give scientists the potential to make them into many different granules so high numbers of DNA sections can be coded for and recognized. Cancerous tumours contain many different types of mutating DNA, so therefore quantum dots can be used to identify different kinds of cancer, reducing the requirement for an invasive biopsy which can be uncomfortable for the patient. Quantum dots are highly resilient against photo bleaching and come in a diverse range of colour shades allowing a great range of cells to be identified . The quantum dots also have high contrast which makes them able to recognize the smallest of tumours, allowing early detection.

Using superparamagnetic iron oxide nanoparticles in order to increase the visual distinction between cancer and normal brain tissue. The nanoprobe is made of up an iron oxide core, coated with PEG (polyethylene glycol), and joined together with the targeting agent chlorotoxin. Chlorotoxin is a scorpion derived 36 amino acid peptide, which has high selectivity for gliomas and other brain cancers, once it has been injected into the bloodstream. It has also been show to be able to bind to medullablastoma, prostatecancer and intestinal cancer. Non targeting nanoparticles are those which do not contain agents which are able to specifically target and bind to the cancer cells, but chlorotoxin is able to bind to cancer cells . This gives the potential to lead on to significant improvements in cancer detection and localization by exploitation of the unique molecular build up of cancer cells.

There is a type of protein called laminin-411 that plays amajor part in the tumour's tendency to create new blood vessels to aid its growth and development and metastasis. The ability of technology to block this protein could lead to an advance in glioma treatment. The single cell organism Physarum polycephalum. It was created with the specific property of being able to permeate the tumour cells, and enter the endosomes. As endosomes mature, they become acidic and the chemical constituent of the drug is able to act at this point and break the endosomes' membranes. The tumour cells' production of laminin-411 is able to be blocked, preventing the protein from being formed and production of new tumour vessels. The drug is able to enter the brain and target the cancer specifically. Due to its strong chemical bonds, the components of the drug are able to avoid damage or separation in tissues or blood plasma during their transport through the human system. The anti tumour component is able to activate directly inside tumour cells, which is different from other nanomedicine drugs that activate at the site of tumour, not in the tumour cells themselves. When the nanobioconjugate has accomplished its tasks, the body digests it completely, leaving no harmful residue. It is changed into carbon dioxide and water which is not toxic to healthy cells. Another advantage is that doesn't stimulate the immune system to produce reactions such as coughs or rashes or any other systems.

\subsection{Interaction of nanoparticles with Collalegen to cure Heart attack}

According to the invention there is provided a synthetic collagen-like material comprising chains of repeating trimeric amino acid building blocks, in which about $30 \%$ of the amino acids are glycine, and at least about $10 \%$ are proline orhydroxyproline, the improvement comprising the incorporation, at least in part, of peptoid residues in said repeating trimeric building blocks and formation of a peptoid residue-containing triple helix from the chains assembled from those peptoid residuecontaining building blocks. invention the synthetic collagen material 
comprises chains of repeating building blocks which are either tripeptide sequences, trimeric dipeptide-peptoid residue sequences or peptide-peptoidresidue-peptide sequences selected from the group consisting of Gly-Xp-Pro; Gly-Pro-Yp; Gly-Pro-Hyp; and Gly-Pro-Pro; or combinations thereof, wherein Xp and Yp are peptoid residues, and the chains have a triple helix conformation similar to that of collagen. In a particularly preferred embodiment, the synthetic collagen material comprises amino acid chains made up of repeating trimeric building blocks selected from the group consisting of Gly-Xp-Pro and Gly-Pro-Yp; or combinations thereof.

\section{CONCLUSION}

It appears that nano drug delivery systems hold great potential to overcome some of the barriers to efficient targeting of cells and molecules in heart valves and cancer. The ability of nanoparticles to specifically target cancerous brain tumors and defective valves of hearts gives a promising possibility of minimising damage to healthy body cells, which is a disadvantage of some current treatment methods such as chemotherapy resulting in a great physical and emotional impact on the patient receiving treatment. Furthermore, the future remains exciting and wide open.

\section{REFERENCE}

1. Won Hyuk Suh, Kenneth S. Suslick, Galen D. Stucky and Yoo-Hun Suh.Nanotechnology nanotoxicology andneuroscience. Prog Neurobiol.2009;87(3):133-170.

2. Xing-Jie Liang, Chunying chen, Yuliang Zhao, Lee Jia and Paul C. Wang.Biopharmaceuticals and therapeutical potentials of engineered nanomaterial.

6. Hagit Bar, Iftach Yacoby and Itai Benhar.Killing cancer cells by targeted drug carrying phage nanomedicines. BMC Biotechnology. 2008;8:37.

9. Nathaniel Huebsch, David J. Mooney. Nature. 2009;462(7272):426-432.

10. Jason H. Sakamoto et al. Enabling individualized therapy through nanotechnology. Pharmacol Res.2010;62(2):57-89.

11. Shihua Jin MD and Vinod Labhasetwar PHD. Nanotechnology in urology. Urol Clin North Am. 2009;36(2):179-188.

12. Suegei V Vinogradov. Polymeric nanogel formulations of nucleoside analogues.Expert Opin Drug Deliv. 2007;4(1):5-17.

14. Michelle Pautler and Sara Brenner.Nanomedicne; promises and challenges for the future of public health.International Journal of Nano medicine.2010;5:803-809.

15. Wim H De Jong and Paul JA Borm. Drug delivery and nanoparticles, applications and hazards. International Journal of Nanomedicine. 2008:3(2):133-149.

16. Mallanagouda Patil, Dhoom Sing Mehta and Sowjanya Guwa. Future impact of nanotechnology on medicine and dentistry. J Indian Soc Periodontol. 2008;12(2):34-40.

17. Mette Ebbesen and Thomas G Jensen.Nanomedicine: Techniques, potentials and ethical implications. Hindawi Publishing Corporation Journal of Biomedicine and Biotechnology. 2006:1-11.

18. Kyle Phua and Kam W Leong. Microscale oral delivery devices incorporating nanoparticles. Nanomedicine (Lond).2010;5(2):161-163.

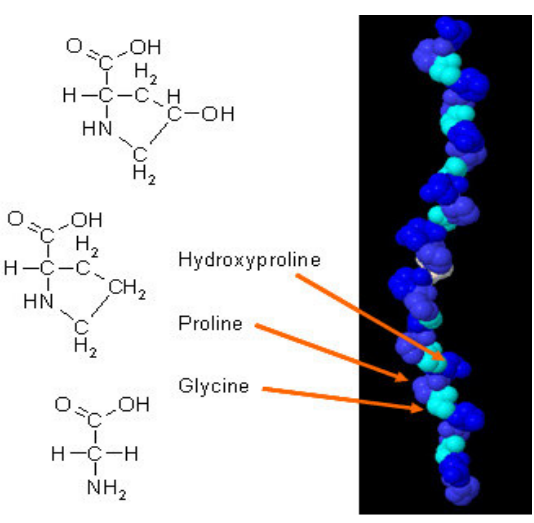

Fig.1 Collalegen

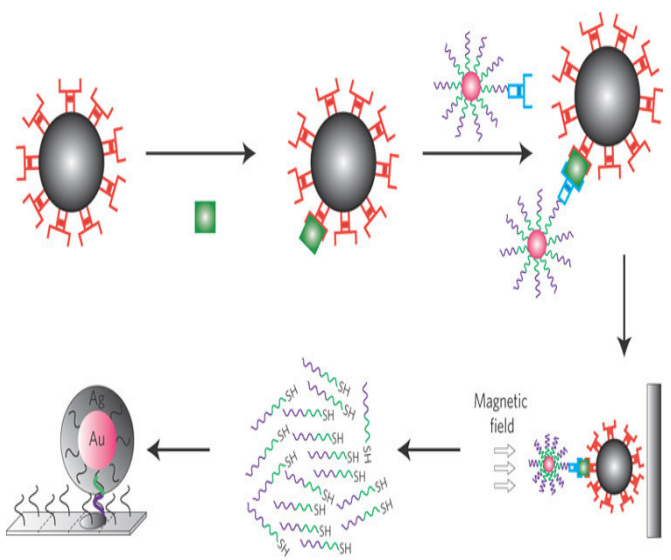

Fig.2 Nanoparticles through ionic gelation 